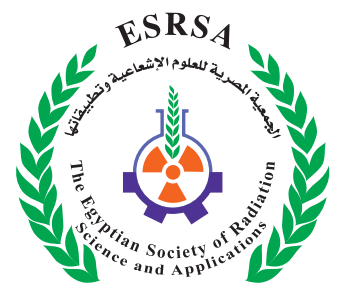

J. Nucl. Tech. Appl. Sci., Vol. 8, PP. 179 : 186 (2020)

\title{
A Study of Water Phantom Homogeneity from DICOM CT-Images Based on Image J
}

Yati, H. ${ }^{1}$; Sra, H.P. ${ }^{1}$; Thareq, B. $^{2}$; Novitrian, N. $^{1}$; Freddy, H. ${ }^{1}$; Abdul, W.

Received: 21/08/2020

Accepted: $15 / 11 / 2020$

DOI: $10.21608 /$ jntas.2020.38220.1028

E.mail:yatihardiyanti@gmail.com

\section{ABSTRACT}

\begin{abstract}
The real data for water phantom have complex variations in the distribution of CT (Computed Tomography) number values for each slice. Thus, the accuracy of dose calculation on a homogeneous medium becomes very important to be reviewed. This study aims to review the distribution of CT numbers (in Hounsfield Units, HU) in a water phantom homogeneity determination. The water phantom was of the IBA dose 1 type, with dimensions of $40 \mathrm{~cm}$ x $35 \mathrm{~cm}$ x $35 \mathrm{~cm}$. The image dimensions were $500 \mathrm{x}$ $500 \mathrm{~mm}^{2}$ with a $1 \mathrm{~mm}$ pixel thickness, and $512 \times 512$ matrix size at image reconstruction. The overall division of water phantom slices was taken from the z-direction or parallel to the patient table into 441 slices. Water phantom homogeneity determination consisted of scanning procedures and image processing. Based on the analysis of the CT number distribution of all slices, the accepted slices were composed of 350 water and 18 acrylic slices. Furthermore, 42 anomaly slices were identified from the calculation of mean and standard deviation. The ROI (Region of Interest) was also considered, reducing water CT number to $53.06 \mathrm{HU}$. Based on this study, a method for homogeneity determination from the distribution of CT numbers in water phantom IBA Dose 1 was developed.

ABSTRACT
\end{abstract}

\author{
Water Phantom, \\ CT Number, $(\mathrm{HU})$ \\ Distribution, \\ Homogeneity, ImageJ. \\ KEYWORDS
}

E.mailyathardiyanti@gmail.com 


\section{INTRODUCTION}

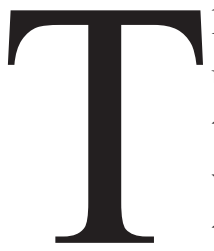

he type of standard water phantom used for therapy needs to be investigated. It is important to justify the water phantom $\mathrm{CT}$ number distribution in water and there is no another material such as air due to the presence of metal artifacts. The real data for water phantom have complex variations in the distribution of $\mathrm{CT}$ number values for each slice. The variation in the $\mathrm{CT}$ number value in each water phantom slice can also affect the absorbed dose (Dobbs et al., 1983, Wong et al., 1983, Brahme, 1984, Dutreix, 1984, Huizenga et al., 1985, Bushong, 2000, Rong et al., 2009, ad Veinot et al., 2016). Thus, the accuracy of dose calculation on a homogeneous medium becomes very important to be reviewed as an essential part of TPS (Treatment Planning System) commissioning.

There are many parameters used to analyze water phantom homogeneity. But in this study, the mean CT number, standard deviation, and the difference between the calculated and measured cross-sectional areas of water phantom were used to analyze CT number distribution and water phantom homogeneity. This study was focused on the CT number and uniformity parameters. Uniformity illustrates how the image in a homogeneous material can be uniformly displayed. The uniformity parameters reported (Goldman et al., 2007) are very important to ensure that cupping and beam-hardening artifacts can be avoided. The process of determining the homogeneity for a water phantom refers to the provisions provided by the American College of Radiology Acceptance Criteria (Papp, 2006). The mean value of $\mathrm{CT}$ number in the water phantom is divided according to three types of materials: polyethylene (between -107 and -87 HU), water (between -7 and $+7 \mathrm{HU}$ ), and acrylic (between +110 and $+130 \mathrm{HU}$ ) (White et al., 1982 and Papp, 2006).

The analysis of CT number distribution and water phantom homogeneity refers to several studies conducted previously by several researchers. QA analysis of Catphan CT 500/504/600 phantom had been verified for the homogeneity determination of CT number distribution (Kristine et al., 2014). Some parameter criteria for determining the homogeneity of $\mathrm{CT}$ numbers distribution consist of low contrast resolution, water CT number, air CT number, image noise, and uniformity or homogeneity. Scanning of the mobile homogeneous phantom was also conducted using cone-beam CT to evaluate the CT number distribution (Siewerdsen et al., 2019). Analysis related to the homogeneity of KPIs (Key Performance Indicators) was introduced by Nowik et al (2015) about increasing the possibility of identifying artifacts that are related to the degradation of CT scanner performance, such as ring artifacts and local non-uniformities caused by air bubbles in the cooling oil. The size of ROI is determined to provide a good tradeoff (artifacts may be undetected) and to avoid errors in the statistical analysis of CT numbers distribution. KPI homogeneity is defined as the maximum difference in the mean CT number between each ROI.

In this study, we have used the mean CT number, standard deviation, and the difference between the calculated and measured cross-sectional areas of water phantom parameters to study the CT number distribution of each slice of water phantom by unchanging the arrangement of the water phantom slice. Thus, the specific purpose of this study was to provide insight to review the distribution of CT numbers (HU) in a water phantom, to determine homogeneity for a water phantom that will contribute to treatment planning.

\section{MATERIALS AND METHODS}

\section{Materials}

In present study, the water phantom utilized during the measurements was the IBA dose 1 type water phantom, with the dimensions of $40 \mathrm{~cm} \mathrm{x} 35 \mathrm{~cm} \mathrm{x}$ $35 \mathrm{~cm}$. 


\section{Methods}

\section{Scanning procedures}

The CT image data of the water phantom were obtained from measurements conducted in one of the private hospitals in Bandung City, West Java Province, Indonesia. The data were obtained using a Siemens SOMATOM Emotion CT scanner device, with the following scan parameters: $130 \mathrm{kVp}$ and 320 $\mathrm{mAs}$ tube voltage, $1 \mathrm{~mm}$ slice thickness, and $512 \mathrm{x}$ 512 pixels image output. The image dimensions are $500 \mathrm{~mm}$ x $500 \mathrm{~mm}$, with a $1 \mathrm{~mm}$ pixel thickness for each slice. The scanning process was conducted in a spiral direction for each single tube rotation. The phantom was scanned from the z-direction or parallel to the patient table, yielding 441 slices that occupied $230 \mathrm{MB}$ of storage space. Output for the CT image data from image reconstruction was stored in DICOM format, using 16-bit pixel precision depth.

\section{Image processing}

The outlined steps for image analysis are shown in Figure 1. In general, the procedure consists of two large parts: analysis of bit depth and analysis of CT number distribution.

\section{- Bit depth analysis}

Bit depth analysis was performed to understand the effect of lower pixel depth and its conversion on CT image. To address this purpose, the default bit depth generated by the scanner was downgraded to 8-bit depth. The bit depth conversion was assumed to reduce storage space; however, the reduction of grayscale intensity that follows the conversion is also known as responsible for a reduction in diagnostic information of CT image (Smith $\boldsymbol{e t}$ al., 1992, Sage et al., 2001 and Nuttawan, 2004) Histogram analysis was performed to evaluate the pixel distribution over the HU range. Since the result was obtained using converted images, histogram adjustment was needed to avoid ambiguity of histogram reading. The number of colors, which was
65536 on a 16-bit image, was adjusted to 256 based on the number of colors that can be represented by an 8-bit image. Since the adjustment would affect bin width value on the histogram of the 8-bit depth image, the bin width value was also adjusted by dividing the range of CT number by the number of colors of the respective bit depth.

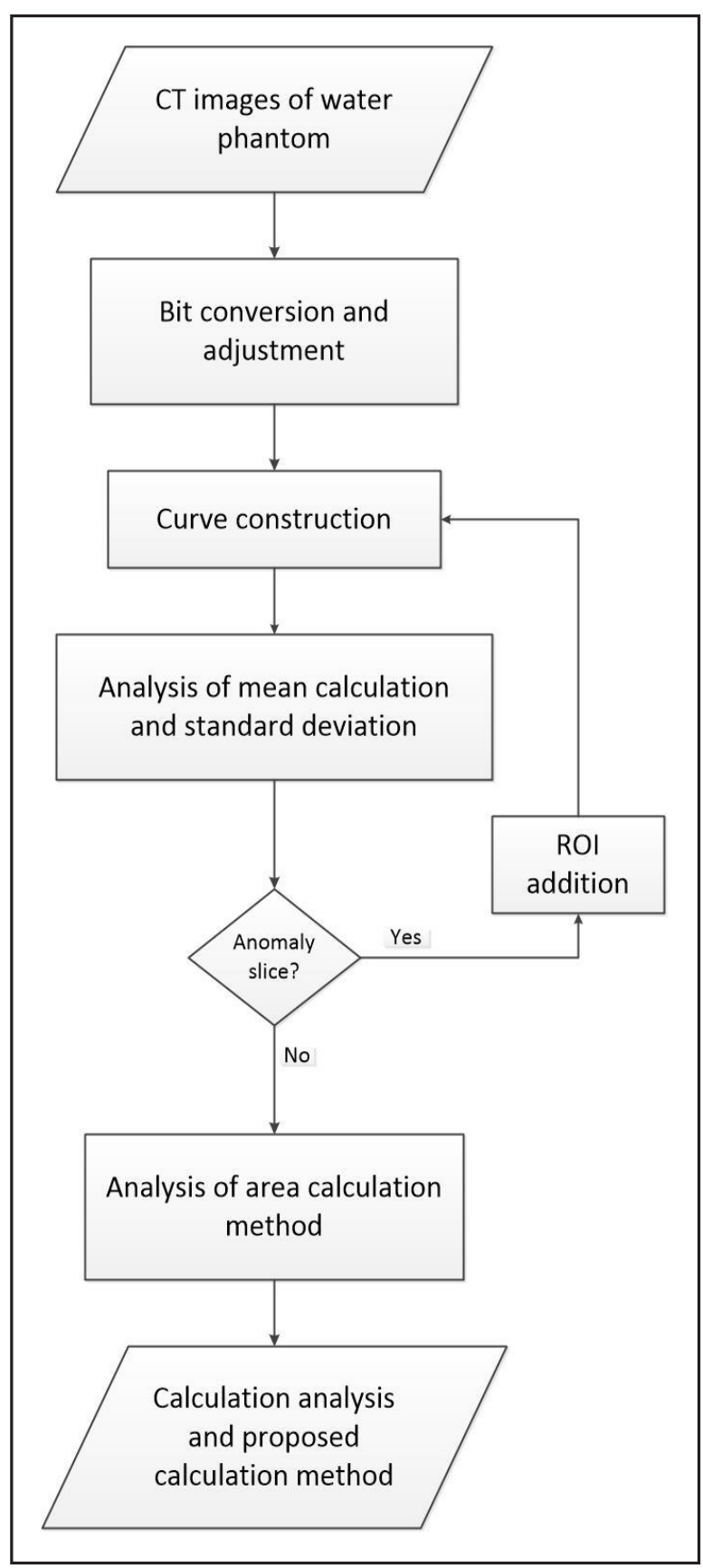

Fig. (1): The flowchart of conducted image analysis. 


\section{- CT number distribution analysis}

The converted CT image then underwent CT number distribution analysis. In this study, ImageJ Java-based image processing program was employed as tool for the observation and analysis of the CT number distribution, which was conducted in several steps. The first step was the construction of pixel distribution curve on the observed range on each slice. The construction was based on the histogram curve reading with 256-bin data distribution; however, the observation was narrowed down to the 110-bin data, which shaped the Gaussian distribution.

The second step was the analysis of calculating the mean and standard deviation (Payne, 2001 and Seeram, 2001). The mean CT number of the water is estimated to be on the range of $-7 \mathrm{HU}$ to $7 \mathrm{HU}$ and the shaped Gaussian curve. The slice in which the mean CT number was configured outside of the range was considered as an anomaly slice. The identification of each anomaly slice was conducted by the addition of Regions of Interest (ROIs) on each image. The mean and standard deviation values before ROI addition were then compared to the values after ROI addition. The variation of maximum and minimum values for $\mathrm{CT}$ number distribution on each slice is affected by the variation of bin width values. Hence, a study on the variation of bin width values is regarded as important to obtain the accuracy of mean CT number calculation.

The third step was the analysis related to the method of area calculation under the normal distribution curve on each slice. It should be noted that only all slices with water content were selected, and each slice was already corrected based on the symmetrical Gaussian curve, focused on the range of -7 $\mathrm{HU}$ to $+7 \mathrm{HU}$, which represents the mean CT number of the water. The area was calculated by Equation 1 . The calculation results on all slices were then averaged.

Area percentage $=\frac{\text { number of pixels under Gaussian curvature }}{\text { number of pixels of slice }} \times 100 \%$
In this study, a method to determine the geometry of the cross-sectional area measurement on the water phantom was developed. The calculation method is described by Equation 2 .

Area percentage $=\frac{\text { cross-sectional size of water phantom }}{\text { size of image slice }} \times 100 \%$

Based on this analysis, the method for geometrical determination of the cross-sectional area for a water phantom was constructed. Therefore, the difference from the comparison of the cross-sectional area for a water phantom can be obtained based on the results of the analysis using measurements and calculations.

\section{RESULTS}

The analysis of CT number distribution was performed for each slice. CT number distribution from each water phantom slice was reviewed using ImageJ in several steps.

\section{Homogeneity determination}

Analyses were conducted for several parameters related to homogeneity, which are mean CT number distribution, standard deviation, and area under the normal distribution curve. Based on the analysis of CT number distribution on all slices, 368 of 441 slices were accepted as the sample slices. The accepted slices were composed of 350 water and 18 acrylic slices. Furthermore, 42 anomaly slices were identified from the calculation of mean and standard deviation on all water slices.

The ROI addition to a selected slice is shown in Figure 2. Slice 27 was chosen and found to have a Gaussian curve on the range from $-7 \mathrm{HU}$ to $7 \mathrm{HU}$. However, slice 27 was observed to be an anomaly slice since it also contained metal artifacts on 3071 $\mathrm{HU}$, which indicates aluminum content, and air on $-1000 \mathrm{HU}$. The slice and histogram comparison of ROI implementation on slice 27 is also presented in Figure 2. 
Slice 27
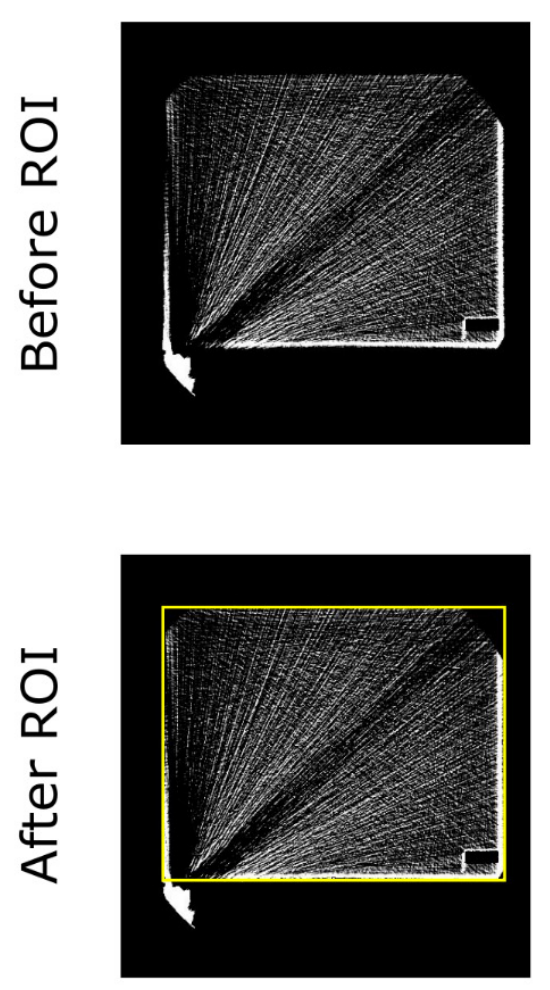

Histogram of slice 27
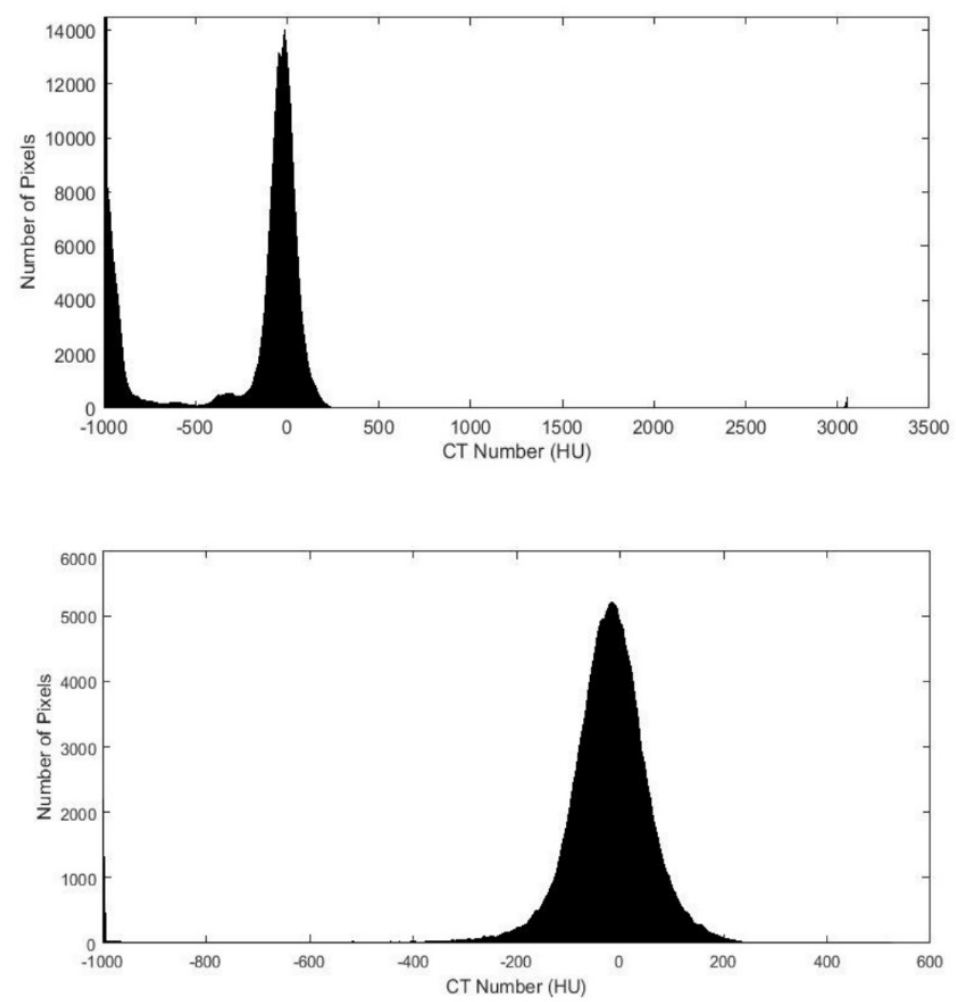

Fig. (2): The comparison of image obtained before and after ROI addition on slice 27. In ImageJ, the ROI was implemented.

The bin width variation was affected by the variation of minimum and maximum values on $\mathrm{CT}$ number distribution. In slice 27 , which was categorized as an anomaly, it had a variation range from $-1000 \mathrm{HU}$ to $3071 \mathrm{HU}$, resulting in a bin width value of 15.9. The ROI addition to this slice changed the minimum and maximum $\mathrm{CT}$ numbers to be -1000 HU and $563 \mathrm{HU}$, respectively. Furthermore, the minimum and maximum bin width values from all accepted slices were found to be 4.95 and 6.11 , respectively.

The distribution of the mean CT number of all slices, with and without ROI addition, was compared to observe the effect of ROI on anomaly slices, as presented in Figure 3. Based on the observation of anomaly slices, the range of $-1000 \mathrm{HU}$ to 477.21 $\mathrm{HU}$ was obtained as the minimum and maximum $\mathrm{CT}$ numbers of all anomaly slices.

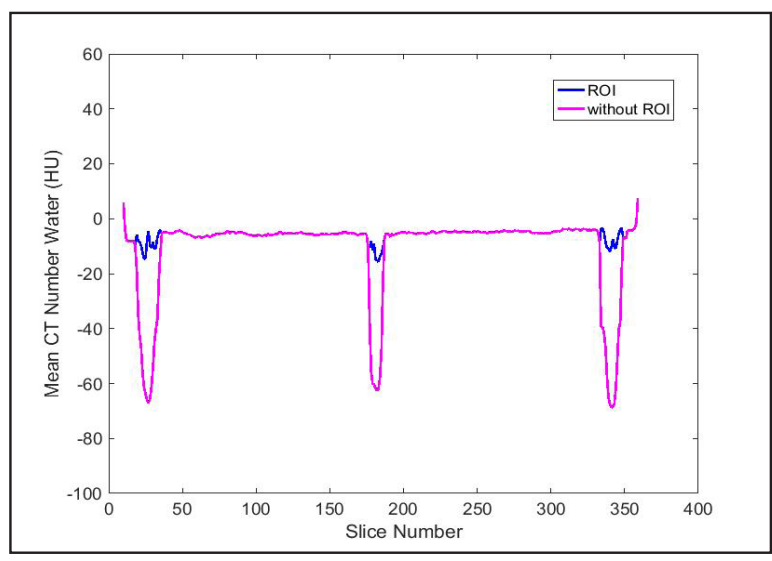

Fig. (3): The mean CT number distribution of water material with and without ROI addition from 350 water phantom slices.

Figure 3 shows that the ROI addition was able to shift the mean CT number distribution of the anomaly slices from the range of $-12.55 \mathrm{HU}$ to -68.76 $\mathrm{HU}$ to a range of $-3.40 \mathrm{HU}$ to $-15.70 \mathrm{HU}$, which is approximate to the range of water compared to the 
analysis before ROI implementation. The ROI was also considered to reduce water HU difference to $53.06 \mathrm{HU}$.

Based on these analyses, the mean CT number distributions of water and acrylic for all corrected slices were calculated at $-5.66 \mathrm{HU}$ and $70.94 \mathrm{HU}$, respectively. The range of slices from 10 to 359 shows the mean CT number distribution for water material. The conformity between calculated and geometric measurements was reached at $35 \mathrm{~cm}$ (350 slices of water phantom).

The distribution of the standard deviation is also different among the mean CT numbers of anomaly slices with and without ROI addition. The standard deviations of the mean CT numbers without ROI addition were calculated on the range of 71.04 to 190.44 on anomaly slices. The ROI addition was observed to decrease the range of 59.24 to 74.37 .

\section{The cross-sectional area of the water phantom}

The results of area calculation under the normal distribution curve are presented in Figure 4. The average of area percentage from all slices was calculated to be $53.87 \%$. Meanwhile, based on the analysis of geometrical dimensions, the comparison of the cross-sectional area of water phantom to CT image data was calculated to be $51.2 \%$. From both results, the difference between the calculation and measurement results of the water phantom cross-sectional area was found to be $2.67 \%$.

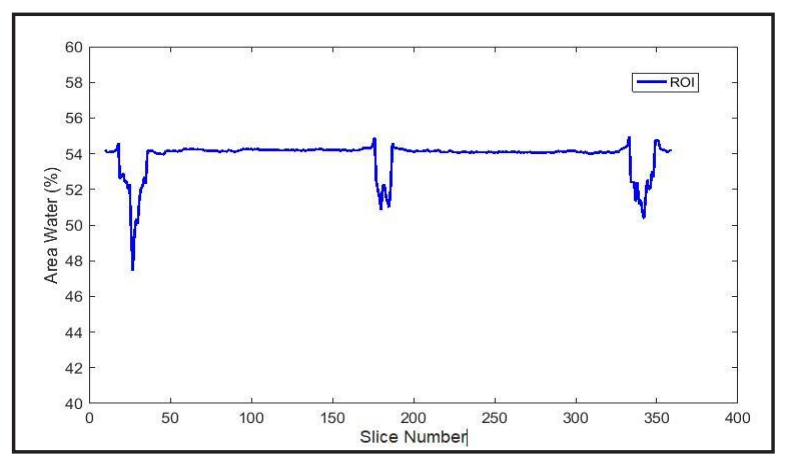

Fig. (4): The average of area percentage under normal distribution curve from 350 slice water phantom.

\section{DISCUSSIONS}

An analysis to determine the homogeneity of water phantom from the distribution of $\mathrm{CT}$ numbers in each slice has been carried out with several proposed parameters, including the mean CT number and standard deviation, in comparison with the criteria presented by (Kristine Gulliksrud et al., 2014). The slices were accepted based on acceptance criteria regarding the distribution of $\mathrm{CT}$ numbers as the basis for taking the next slice limits to be tested. In a previous study, analysis of QA characteristics on Catphan 500/504/600 CT phantoms was carried out by several researchers concerning low-contrast resolution, CT numbers of water, CT numbers of air, image noise, and uniformity or homogeneity. (Kristine Gulliksrud et al., 2014) compared manual image analysis of the distribution of CT numbers from the DicomWorks 1.3.5 and ImageJ 1.45s image viewers with results obtained from the QAlite automatic evaluation software. Whereas, in this paper, the images were analyzed manually from the distribution of CT numbers with the ImageJ 1.50i image viewer program (Wayne Rasband National Institutes of Health, USA).

Based on the results of the analysis with Image J on DICOM data for each range of slices, greyscale image changes in the form of black files (air) in the water phantom were found. Upon review, the black files (air) show the presence of metal artifacts that arise due to the aluminum metal buffer on the three feet of the water phantom.

Thus, giving an ROI parameter that is adjusted to the geometry size of the water phantom is very important to be applied for slice anomalies, which allows the bin width variation of the CT number distribution to focus on the water material.

In this study, the important point presented is that if the determined homogeneity encounter constraints related to the mean CT number in each slice does not meet the range given by the Acceptance 
Criteria from the American College of Radiology (Papp, 2006), it is necessary to identify the KPI (key performance indicator) homogeneity.

Several options need to be considered by the user. This includes the following possibilities: checking the input data in the form of a CT number distribution from the $\mathrm{CT}$ image data in each slice with ImageJ; checking the settings in the measurement test; checking the overall water phantom geometry size according to the provision of the ROI parameter with the geometry size of the water phantom to focus on the distribution of CT numbers for water material; checking the calculated bin width value after giving the ROI parameter; limiting taken bin observations for each slice that has been given an ROI parameter; and checking the geometry size of the water phantom and comparing the measurement results with the calculation of the CT image data size for each slice.

The above refers to previous research by Nowik et al. (2015) regarding the homogeneity of KPIs that are introduced to increase the possibility of identifying artifacts that are related to degradation of CT scanner performance (such as ring artifacts and local non-uniformities caused by air bubbles in cooling oil). The size of ROI is determined to provide a good trade-off (artifacts may not be detected) and to avoid errors in the statistical analysis of the distribution of CT numbers. KPI homogeneity is defined as the maximum difference in the mean $\mathrm{CT}$ number between each ROI.

The newness is related to the method of determining the geometry of cross-sectional areas for water phantoms. In other words, as the difference in the size of the water phantom cross-sectional area measured by the results of the calculation becomes smaller, it is expected that the dose absorbed becomes more accurate, but still below the safety standard of $<3 \%$. The method gives an initial prediction of the studied water phantom, showing that the water phantom can be regarded to have the criteria for ideal form.

\section{CONCLUSIONS}

A homogeneity determination method from the distribution of CT numbers or HU for water phantoms with Image J was reviewed. The material homogeneity determination for water phantom has been successfully displayed based on statistical analysis. The calculation method was relatively valid. The difference between the calculated and measured crosssectional areas of water phantom was found to be $2.67 \%$. This can affect the dose absorbed variation but is still below the safe standard of $<3 \%$. Thus, the accuracy of dose calculation on a homogeneous medium can be obtained. The protocol standard in this analysis can be used to determine the homogeneity of water phantoms.

This study can be used as a reference for analyzing the dose distribution using TPS (Treatment Planning System) in commissioning tests. So, further studies may be conducted within the area of dose distribution analysis using other methods in the near future.

\section{REFERENCES}

- Brahme, A. (1984): Dosimetric precision requirements in radiation therapy. Acta Radiol Oncol., 23: 379.

- Bushong, S.C. (2000): Computed Tomography: Essential of Medical Imaging Series. New York: The MacGraw-Hill Companies.

- Dobbs, H.J.; Parker, R.P.; Hodson, N.J.; Hobday, P. and Husband, J.E. (1983): The use of CT in radiotherapy treatment planning. Radiother. Oncol., 1(2): 133.

- Dutreix, A. (1984): When and how can we improve precision in radiotherapy?. Radiother. Oncol., 2: 275.

- Goldman, L.W. (2007): Principles of CT: Radiation Dose and Image Quality. J. Nuc. Med. Tech., 35: 213.

- Huizenga, H. and Storchi P.R.M. (1985): The Use of Computed Tomography Number Dose Calcula- 
tions for Radiation Therapy. Acta Radiol. Oncol., 24(6): 509.

- Kristine, G.; Caroline, S. and Anne, C.T.M. (2014): How to measure CT image quality: Variations in CTnumbers, uniformity and low contrast resolution for a CT quality assurance phantom. European J. Med. Phy., 30(4): 521.

- Nowik, P.; Bujila, R.; Polundniowski, G. and Fransson, A. (2015): Quality control of CT systems by automated monitoring of key performance indicators: a two-year study. J. App. Clin. Med. Phys., 16(4): 254.

- Nuttawan, J. (2004): CT Protocol. Bangkok: Radiology Departement Of Takshin Hospital.

- Papp, J. (2006): Quality Management in The Imajing Sciences. $3^{\text {rd }}$ ed. Missoouri: Mosby Elsevier, Inc.

- Payne, T. (2001): Fundamental Principles of CT Performance Evaluation AAPM Summer School. Minneapolis: Abbott Northwestern Hospital.

- Rong, Y.; Smilowitz, J.; Tewatia, D. and Tomé, W.A. (2009): Dose Calculation On Kv Cone Beam Ct Images: An Investigation Of The Hu-Density Conversion Stability And Dose, Accuracy Using The SiteSpecific Calibratio, Medical Dosimetry: official $J$. Am. Ass. Med. Dos., 35(3): 195.

- Sage, D. and Unser, M. (2001): Proc of the 2001 IEEE International Conference on Image Processing. Thessaloniki, 3: 298.

- Seeram, E. (2001): Computed Tomography: Physical Principles, Clinical Applications, and Quality Control. $2^{\text {rd }}$ ed. Canada: W. B. Sounders Company.

- Siewerdsen, J.H.; Uneri, A.; Hernandez, A.M.; Burkett, G.W. and Boone, J.M. (2019): Cone-beam CT dose and imaging performance evaluation with a modular, multipurpose phantom. Med. Phys., 47(2): 467.

- Smith, H.J.; Bakke, S.J.; Smevik, B.; Hald, J.K.; Moen, G.; Rudenhed, B. and Abildgaard, A.
(1992): Comparison of 12-Bit and 8-Bit Gray Scale Resolution in MR Imaging of the CNS, Acta Radiol.,33(6): 505 .

- Veinot, K.G.; Eckerman, K.F. and Hertel, N.E. (2016): Organ and effective dose coefficients for cranial and caudal irradiation geometries: photons. Rad. Prot. Dos., 168(2): 167.

- White, D.R. and Constantinou C. (1982): Anthropomorphic phantom materials. Med. Rad. Phys., 1: 133.

- Wong, J.W and Henkelman R.M. (1983): A new approach to CT pixel-based photon dose calculations in heterogeneous media. Med. Phys., 10: 199. 